\title{
Kinematic Reduction and Planning using Symmetry for a Variable Inertia Mechanical System
}

\author{
Ravi Balasubramanian and Alfred A. Rizzi \\ \{bravi, arizzi\}@cs.cmu.edu \\ The Robotics Institute, Carnegie Mellon University, \\ Pittsburgh, PA 15213, USA.
}

\begin{abstract}
Motivated by finding locomotion primitives for a legged robot, we present controllability results and kinematic reduction for a variable inertia mechanical system. We show that the mechanical system is configuration controllable and use the symmetry resulting from angular momentum conservation to develop a kinematic representation of the mechanical system. We also show through simulation how plans for the kinematic representation can be implemented on the full dynamical mechanical system. Our hope is that this technique will lead us to a general procedure for solving the gait synthesis problem.
\end{abstract}

\section{INTRODUCTION}

Planning for mechanical systems can be difficult, since such systems typically have velocity-related drift vector fields and input controls which are generalized forces (comparable to acceleration inputs). In contrast, planning for kinematic systems is easier, since they do not have drift vector fields and have input controls which are velocities [8][9]. Thus, if a mechanical system can be reduced to a kinematic system, then we will be able to plan the motion for the kinematic system and cause the mechanical system to track those velocity inputs through careful use of the force inputs. Unfortunately, short of just integrating the system equations (a difficult process), there is no systematic procedure for finding such a kinematic reduction. This paper presents controllability results [12] and kinematic reduction [11][7] using the system's symmetry for a simple mechanical system called the Yaw model (see Fig. 1).

The Yaw model is a simplification of Rocking and Rolling Robot (RRRobot) (see Fig. 2), a highcentered robot that locomotes using the dynamic effect of swinging its legs; this locomotion, termed legless locomotion [2][3], is a result of the interaction between RRRobot body attitude oscillations and the nonholonomic contact constraints. In [4], we presented three leg motion patterns, or gaits, that locomote RRRobot in different directions in the plane and also studied simplified models where the effect of the contact constraints on the body attitude

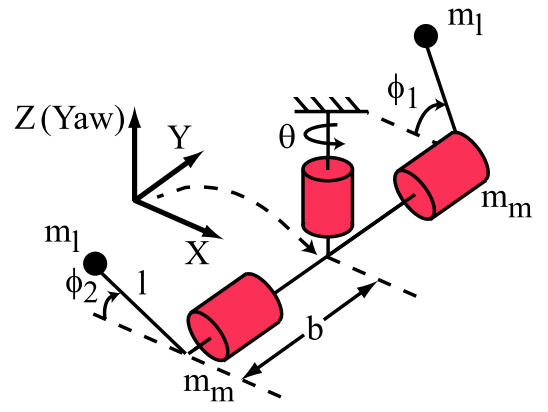

Fig. 1. The Yaw Model: the body can freely rotate about the yaw axis, and the two legs with point masses at the distal ends are actuated.

dynamics analysis is ignored. But we still are not able to find a systematic technique for finding gaits inducing controlled body attitude changes; that is, given a goal RRRobot attitude trajectory, we do not have a procedure for finding leg trajectories that generate the desired body rotations. To understand the relationship between RRRobot body rotations and leg motions, we consider simplified models such as the Yaw model where we can study the relationship between leg motions and specific body rotational freedoms (see [3] and [4] for other simplified models). This paper relates body yaw to specific gaits and presents a path planning procedure for the Yaw model. Understanding these simple models may help find a path planning strategy for RRRobot or inspire other legless locomotion designs.

The Yaw model has three degrees of freedomthe body is free to rotate about the yaw axis, and each massless leg has a single-actuated hip joint and a point mass at its distal end. An important aspect of the Yaw model is that its inertia about the yaw axis changes with leg configuration but is invariant to yaw rotations. In Balasubramanian et al. [2][3], we show how cyclic interleaved leg motions exploit the variable yaw inertia to produce net yaw.

After presenting some background material in Section II, we show that the Yaw model is configuration 


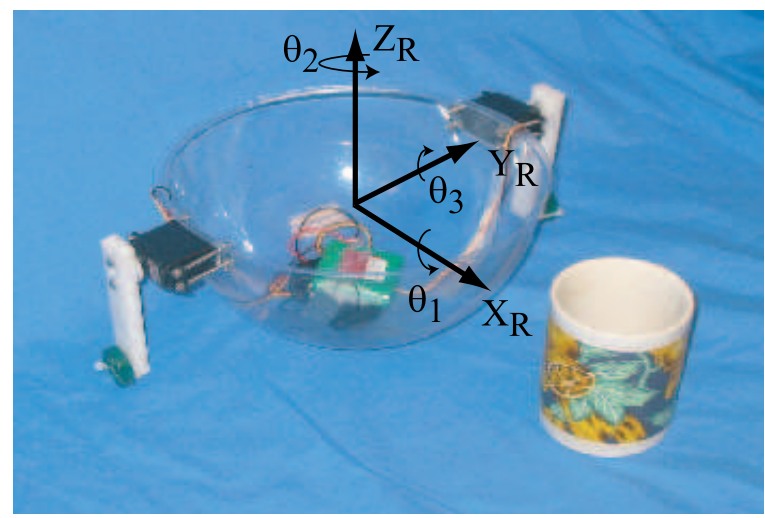

Fig. 2. Rocking and Rolling Robot locomotes in the plane using the dynamic effect of swinging its legs.

controllable and present a kinematic representation of the Yaw model in Section III. In Section IV, we show through simulation how plans for the kinematic representation can be implemented on the mechanical system.

\section{BACKGROUND}

Lewis and Murray [13] provide a foundation for studying simple mechanical systems whose Lagrangian is the system kinetic energy minus the potential energy; we will consider only systems with constant potential energy. The equations of motion for a mechanical system $S_{D}$ with an $n$-dimensional configuration space $Q$ and a Riemannian metric $g$ : $T Q \rightarrow \mathbb{R}$ can be expressed as

$$
\nabla_{\dot{c}(t)} \dot{c}(t)=u^{a}(t) Y_{a}(c(t))
$$

where $c(t)=\left(q^{1}(t), \ldots, q^{n}(t)\right)$ is a curve on $Q, \nabla$ is an arbitrary affine connection defined by

$$
\nabla_{X} Y=\left(\frac{\partial Y^{i}}{\partial q^{j}} X^{j}+\Gamma_{j k}^{i} X^{j} Y^{k}\right) \frac{\partial}{\partial q^{i}}
$$

for a set of Christoffel symbols $\Gamma_{j k}^{i}, u^{a} \in \mathbb{R}$ are the inputs, $Y_{1}, \ldots, Y_{m}$ are the control vector fields, $X \in T_{q} Q$ is a vector field, and we use the Einstein summation notation, where we sum over all possible values of indices which occur twice in a single term. For example,

$$
u^{a}(t) Y_{a}(c(t))=\sum_{a=1}^{m} u_{a}(t) Y_{a}(c(t)) .
$$

For those unfamiliar with affine and Riemannian differential geometry, we suggest Kobayashi and Nomizu [14]; we will use the notation of Abraham and Marsden [1] in this paper.
Defi nition 1 Given an affine connection $\nabla$ on $Q$, the symmetric product is defined as

$$
\langle X: Y\rangle=\nabla_{X} Y+\nabla_{Y} X, \forall X, Y \in T Q .
$$

An important property for control systems is the notion of local configuration accessibility, the ability to reach an open set of configurations. Lewis and Murray [13] give the sufficient condition for local configuration accessibility for $S_{D}$. Let $D_{d y n}=$ $\operatorname{Span}\left\{Y_{1}, \ldots, Y_{m}\right\}$ be the input distribution for $S_{D}$, $\overline{\operatorname{Sym}}\left(D_{d y n}\right)$ be the symmetric closure of $D_{d y n} \subset T Q$, and $\overline{L i e}\left(D_{d y n}\right)$ be the involutive closure of $D_{d y n}$.

Theorem 1 [13] The mechanical system $S_{D}$ is locally configuration accessible at $q$ if $\overline{\operatorname{Lie}}\left(\overline{\operatorname{Sym}}\left(D_{d y n}\right)\right)_{q}=$ $T_{q} Q$.

Using Theorem 1, Lewis and Murray show that a rigid body in the plane is locally configuration accessible with two inputs; one of the inputs is a force, and the other can be an off-centered force or a torque. Thus, with these two inputs, the rigid body can reach an open set of local configurations.

Before we state the sufficient condition for Small Time Local Configuration Controllability (STLCC) of mechanical systems, the ability to reach a local neighborhood of configurations, we define the notion of good and bad symmetric products. A symmetric product is bad if it contains an even number of each of the vector fields $Y_{a}, a=1, \ldots, m$; otherwise a symmetric product is good. Lewis [12] gives the sufficient condition for STLCC:

Theorem 2 [12] If $\operatorname{Dim}\left(\overline{\operatorname{Lie}}\left(\overline{\operatorname{Sym}}\left(D_{d y n}\right)\right)_{q}\right)=n$, and every bad symmetric product at $q \in Q$ is an $\mathbb{R}$-linear combination of good products of lower degree at $q$, then $S_{D}$ is STLCC at q.

Lewis [12] proves configuration controllability for the snakeboard [10], a variation of the skateboard where the wheel directions can be changed and there is a rotor; that is, with input torques for the wheels and rotor, the snakeboard can reach a local neighborhood of configurations.

Configuration controllability is useful, but still planning for mechanical systems is difficult compared to planning for kinematic models. A driftless kinematic model $S_{K}$ with an $n$-dimensional configuration space $Q$ is defined by the set of linearly independent vector fields $\left\{X_{1}, \ldots, X_{\tilde{m}}\right\}$ such that

$$
\dot{q}(t)=\tilde{u}^{\alpha}(t) X_{\alpha}(c(t)),
$$


where $q \in Q$ and $\tilde{u}^{\alpha} \in \mathbb{R}$. Bullo et. al [6] look at conditions when a mechanical system can be represented by a kinematic model. Let $(q, \tilde{u})$ be a solution of (4) for controls $\tilde{u} \in U_{k i n}$, and $D_{k i n}=\operatorname{Span}\left\{X_{1}, \ldots, X_{\tilde{m}}\right\}$ be the input distribution for the kinematic model. The kinematic model $S_{K}$ is said to be a kinematic reduction of $S_{D}$ if there exist controls $u \in U_{d y n}$ so that $(q, u)$ is a solution of (1). Note that $U_{d y n}$ is not necessarily equal to $U_{k i n}$. This relationship between $D_{k i n}$ and $D_{d y n}$ is summarized as:

Theorem 3 [6] The kinematic model $S_{K}$ is a kinematic reduction of $S_{D}$ if and only if the distribution generated by the vector fields $\left\{X_{i},\left\langle X_{j}: X_{k}\right\rangle \mid i, j, k \in\right.$ $\{1, \ldots, \tilde{m}\}\}$ is a constant rank subbundle of the input distribution $D_{d y n}$.

In addition to the condition placed by Theorem 3 on $D_{d y n}$ and $D_{k i n}, U_{k i n}$ must be such that there exists $u \in U_{d y n}$ that can track the trajectory; for example, $U_{\text {kin }}$ must at least be $C^{2}$ continuous. Lewis [11] shows that the upright rolling disk is kinematically reducible; that is, we can ignore the dynamic effects of inertia while planning paths for the upright rolling disk. This greatly simplifies the planning problem; if we find smooth plans for the kinematic model, those trajectories can be tracked by the dynamic system.

Typically, it is not straightforward to find a kinematic model for a mechanical system. For a generic mechanical system to admit a kinematic reduction, the system must satisfy certain properties as expressed in Theorem 3. An important property of the Yaw model that will help us derive its kinematic reduction is that its Lagrangian is invariant to yaw rotations, that is, there is a symmetry. For the Yaw model, by Noether's theorem [5], this means that the momentum about the yaw axis is conserved in the absence of external forces. In III-C, we will use this symmetry to derive the kinematic reduction for the Yaw model.

\section{THE YAW MOdEL}

The Yaw model body is pivoted at its body center and has two masses, each $m_{m}$, at its ends (see Fig. 1). Each massless leg has an actuated hip joint and a point mass $m_{l}$ at the distal end. The Yaw model configuration is represented by $q=\left(\theta_{2}, \phi_{1}, \phi_{2}\right)^{T} \in Q=$ $\mathbb{S}^{1} \times \mathbb{S}^{1} \times \mathbb{S}^{1}$, where $\theta$ denotes the body configuration, $\phi_{1}$ leg 1's joint configuration, and $\phi_{2}$ leg 2's joint configuration. There is no gravity, there are no joint limits, and torques $u_{1}$ and $u_{2}$ can be applied at leg joints 1 and 2. The Riemannian metric $g$ associated with the Yaw model on $Q$ is

$$
g(q)=\left(\begin{array}{lll}
g_{11} & g_{12} & g_{13} \\
g_{21} & g_{22} & g_{23} \\
g_{31} & g_{32} & g_{33}
\end{array}\right)
$$

where

$g_{11}=2\left(m_{m}+m_{l}\right) b^{2}+m_{l} l^{2}+\frac{1}{2} m_{l} l^{2}\left(\cos 2 \phi_{1}+\right.$ $\left.\left.\cos 2 \phi_{2}\right)\right)$,

$g_{12}=-m_{l} l b \sin \phi_{1}$,

$g_{13}=m_{l} l b \sin \phi_{2}$,

$g_{21}=-m_{l} l b \sin \phi_{1}$,

$g_{22}=m_{l} l^{2}$,

$g_{23}=0$,

$g_{31}=m_{l} l b \sin \phi_{2}$,

$g_{32}=0$,

$g_{33}=m_{l} l^{2}$,

and the twenty seven Christoffel symbols $\Gamma_{j k}^{i}$ are computed as

$$
\Gamma_{j k}^{i}=\frac{1}{2} g^{i l}\left(\frac{\partial g_{j l}}{\partial q^{k}}+\frac{\partial g_{k l}}{\partial q^{j}}-\frac{\partial g_{j k}}{\partial q^{l}}\right),
$$

where $g^{i j}$ are the components of the inverse of $g_{i j}$. Note that there is a symmetry in the Yaw model: $g$ is independent of yaw rotations, that is, $g$ does not depend on $\theta$.

The Yaw model equations of motion are

$$
\ddot{q}^{i}+\Gamma_{j k}^{i} \dot{q}^{j} \dot{q}^{k}=u^{a} Y_{a}^{i}
$$

where $Y_{1}=g^{-1}\left(\begin{array}{l}0 \\ 1 \\ 0\end{array}\right)$, and $Y_{2}=g^{-1}\left(\begin{array}{l}0 \\ 0 \\ 1\end{array}\right)$ are the control vector fields, and $D_{d y n}=\operatorname{Span}\left\{Y_{1}, Y_{2}\right\}$. Row 1 of (7) indicates that the body is not directly actuated; assuming zero system initial velocity, then the body must be stationary when the legs are stationary. Planning system trajectories using (7) is difficult, because of the velocity-related terms and the torque inputs; that is, there is no systematic analytic procedure to find torque inputs to achieve a given goal trajectory. We will see in Section IV how to plan for the Yaw model using a kinematic representation.

\section{A. Configuration Accessibility}

Since $\left\langle Y_{1}: Y_{1}\right\rangle,\left\langle Y_{2}: Y_{2}\right\rangle,\left\langle Y_{1}: Y_{2}\right\rangle \in D_{d y n}$, $\overline{\operatorname{Sym}}\left(D_{d y n}\right)=D_{d y n}$. Except at $\sin \phi_{1}=\sin \phi_{2}=0$, $\operatorname{Rank}\left(\left\{Y_{1}, Y_{2},\left[Y_{1},\left[Y_{1}, Y_{2}\right]\right]\right\}\right)=3$. Thus, by Theorem 1 , $\overline{\operatorname{Lie}}\left(\overline{\operatorname{Sym}}\left(D_{d y n}\right)\right)=T_{q} Q$, and the Yaw model is locally configuration accessible everywhere except when both legs are horizontal. From now on, we will consider only non-singular configurations. Thus, starting from rest at a given configuration, the Yaw model can reach an open set of configurations. 


\section{B. Configuration Controllability}

In III-A, we showed that the Yaw model is configuration accessible. The bad symmetric products $\left\langle Y_{1}: Y_{1}\right\rangle$ and $\left\langle Y_{2}: Y_{2}\right\rangle$ are in the span of $D_{d y n}$. Thus, by Theorem 2, the Yaw model is STLCC, and, starting from rest at a given configuration, the Yaw model can reach a local neighborhood of the initial configuration.

\section{Kinematic Representation}

Using (7), we now derive a kinematic representation of the Yaw model. Note that row 1 of (7) is integrable; that is, if the initial system velocity is zero, we can integrate row 1 to get

$$
g_{11} \dot{\theta}+g_{12} \dot{\phi}_{1}+g_{13} \dot{\phi}_{2}=0
$$

The terms on the left side of (8) add up to the system's yaw momentum; thus, the yaw momentum is always zero.

Using (8), we define the kinematic representation of the Yaw model to be

$$
\dot{q}=X_{1} \tilde{u}_{1}+X_{2} \tilde{u}_{2},
$$

where $X_{1}=\left(\begin{array}{c}-g_{12} / g_{11} \\ 1 \\ 0\end{array}\right), X_{2}=\left(\begin{array}{c}-g_{13} / g_{11} \\ 0 \\ 1\end{array}\right)$, $D_{\text {kin }}=\operatorname{Span}\left\{X_{1}, X_{2}\right\}, \tilde{u}_{a} \in \mathbb{R}$, and we have assumed that we have direct control over the leg joint velocities. Note that the kinematic representation is also invariant to yaw rotations.

\section{Kinematic Reducibility}

Using linear algebra, we can verify that the vector fields $X_{1}$ and $X_{2}$ are in the span of $D_{d y n}$. Also, the symmetric products $\nabla_{X_{i}} X_{j}, i, j=1,2$ are in the span of $D_{d y n}$. Thus, by Theorem 3, the Yaw model is reducible to the kinematic representation in (9), while ensuring $C^{2}$ continuous inputs for the kinematic representation. Note that (9) is much simpler than (7), but represents all the properties of (7). We have, thus, reduced planning for the mechanical system in (7) to a nonholonomic kinematic path planning problem for (9).

Further, we notice that if only one leg moves and the other is fixed, (8) can be integrated again. This induces a holonomic constraint on the Yaw model; that is, the yaw configuration is specified just by the leg configuration and is independent of the path taken by the leg. Suppose, leg 2 is kept fixed at $\phi_{2}$, and

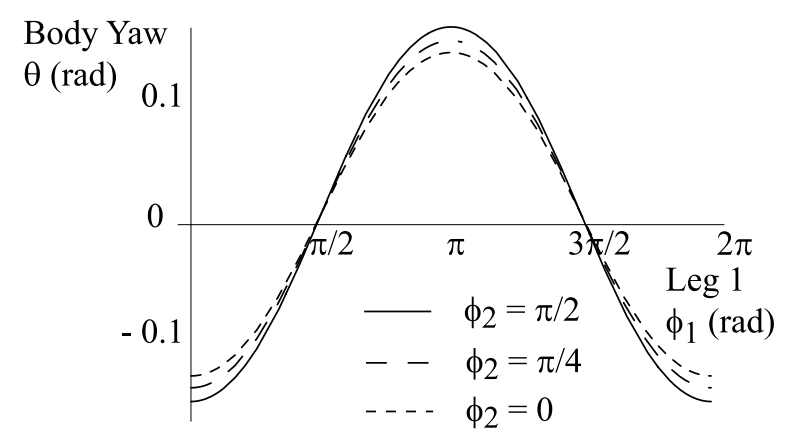

Fig. 3. Body yaw when leg 2 is fi xed and leg 1 is rotated.

leg 1 is moved by $\Delta \phi_{1}$ from $\pi / 2$, the net body yaw is calculated using the holonomic constraint

$$
\Delta \theta=\frac{2 m_{l} l b \operatorname{arctanh}\left(a\left(\Delta \phi_{1}\right)\right)}{\sqrt{m_{l} l^{2} \gamma\left(\phi_{2}\right)}},
$$

where $\gamma\left(\phi_{2}\right)=\left(4 m_{l} l^{2}-2\left(4 m_{l} b^{2}+3 m_{l} l^{2}+\right.\right.$ $\left.\left.4 m_{m} b^{2}+m_{l} l^{2} \cos 2 \phi_{2}\right)\right)$, and $a\left(\Delta \phi_{1}\right)=$ $-2 \sqrt{m_{l}^{2}{ }^{2}} \sin \left(\Delta \phi_{1}\right) / \gamma\left(\phi_{2}\right)$. Thus, when leg 1 is moved to $\phi_{1}=0$, the body rotates by an angle $\Delta \theta=\frac{2 m_{l} l b \operatorname{arctanh}\left(2 \sqrt{m_{l} l^{2}} / \gamma\left(\phi_{2}\right)\right)}{\sqrt{m_{l} l^{2} \gamma\left(\phi_{2}\right)}}$. Fig. 3 shows how the body yaws, as a function of leg 2 configuration, when leg 1 is moved. Similarly, if leg 1 is kept fixed at $\phi_{1}$, and leg 2 is moved by $\Delta \phi_{2}$ from $\pi / 2$, the net body yaw is computed using the holonomic constraint:

$$
\Delta \theta=-\frac{2 m_{l} l b \operatorname{arctanh}\left(a\left(\Delta \phi_{2}\right)\right)}{\sqrt{m_{l} l^{2} \gamma\left(\phi_{1}\right)}}
$$

where $\gamma\left(\phi_{1}\right)=\left(4 m_{l} l^{2}-2\left(4 m_{l} b^{2}+3 m_{l} l^{2}+\right.\right.$ $\left.\left.4 m_{m} b^{2}+m_{l} l^{2} \cos 2 \phi_{1}\right)\right)$, and $a\left(\Delta \phi_{2}\right)=$ $-2 \sqrt{m_{l} l^{2}} \sin \left(\Delta \phi_{2}\right) / \gamma\left(\phi_{1}\right)$.

\section{Z Model Motion Planning}

The Yaw model kinematic reduction in III-C is simple to plan paths for, since we require the following two leg motion patterns or gaits only, one a sinusoidal trajectory and the other a cubic spline trajectory.

- Gait 1: Move the legs simultaneously with outof-phase velocities; for example, $\dot{\phi}_{1}=A \sin t$, and $\dot{\phi}_{2}=A \cos t$ for some suitable amplitude $A$. To ensure that the dynamic inputs can track these velocities, an envelope function is used at the start and end of each cycle to guarantee smoothness in velocity space (see II for conditions on the kinematic model input space).

- Gait 2: Move only one leg, say, using a cubic spline with zero initial and final velocities; keep the other leg stationary. 
Each cycle of Gait 1 produces net body yaw due to the varying mass matrix (see [3] for intuitive thought experiments on net yaw produced by interleaved leg motions). Gait 2 produces body yaw, but if the leg returns to the start configuration, then the net body $\mathrm{Z}$ rotation is zero (see III-D for more details).

Here is a motion planning algorithm for the Yaw model. Without loss of generality, we will assume that the legs start from the legs-up configuration. Let the goal configuration be $q_{g}=\left(\theta_{g}, \phi_{1 g}, \phi_{2 g}\right)$.

1) Precompute body yaw $\alpha$ using (10) when moving leg 1 from $\pi / 2$ to $\phi_{1 g}$ using Gait 2 while keeping leg 2 fixed at $\pi / 2$. Similarly, precompute body yaw $\beta$ using (11) when moving leg 2 from $\pi / 2$ to $\phi_{2 g}$ using Gait 2 while keeping leg 1 fixed at $\phi_{1 g}$. We can compute $\alpha$ and $\beta$ a priori because the Yaw model is invariant to yaw rotations.

2) Choose amplitude $A$ so that the net yaw $\rho$ in one cycle of Gait 1 is sufficiently small to make $c=\left(\theta_{g}-(\alpha+\beta)\right) / \rho$ an integer. Repeat Gait 1 $c$ times.

3) Move leg 1 to $\phi_{1 g}$ using Gait 2. Move leg 2 to $\phi_{2 g}$ using Gait 2 .

Once we compute the trajectories in the kinematic representation for each leg to achieve $q_{g}$, we can track those trajectories in the mechanical system using a simple PD controller.

\section{A. Numerical Results}

The start configuration $q_{s}=(0, \pi / 2, \pi / 2)$ is the legs-up configuration. Let $m_{m}=1.0 \mathrm{~kg}, m_{l}=0.5 \mathrm{~kg}$, $b=0.5 \mathrm{~m}, l=0.5 \mathrm{~m}, A=0.516$, and $q_{g}=$ $(-4 \pi / 180,4 \pi / 9,4 \pi / 9)$ (four degrees body yaw and ten degrees off the legs-up configuration). For Gait 1 , we set the leg velocities as follows:

$$
\begin{aligned}
& \dot{\phi}_{1}=f(t) A \sin (t), \\
& \dot{\phi}_{2}=f(t) A \cos (t),
\end{aligned}
$$

where

$$
f(t)= \begin{cases}1-\mathrm{e}^{-k \bmod (t, 2 \pi)} & \bmod (t, 2 \pi) \leq \pi, \\ 1-\mathrm{e}^{-k(2 \pi-\bmod (t, 2 \pi))} & \text { otherwise, }\end{cases}
$$

and $k=100$. The envelope function ensures that the legs's initial and final velocities during each cycle of Gait 1 are zero. Following the algorithm, we precompute $\alpha=-0.02889$ and $\beta=0.02875$. With $\rho=-0.014$, we repeat Gait 2 five times so that the body reaches the required yaw configuration. Now, when the legs are moved one by one to their goal positions using Gait 2, the Yaw model will reach $q_{g}$. Fig. 4 shows the time history of body rotation and

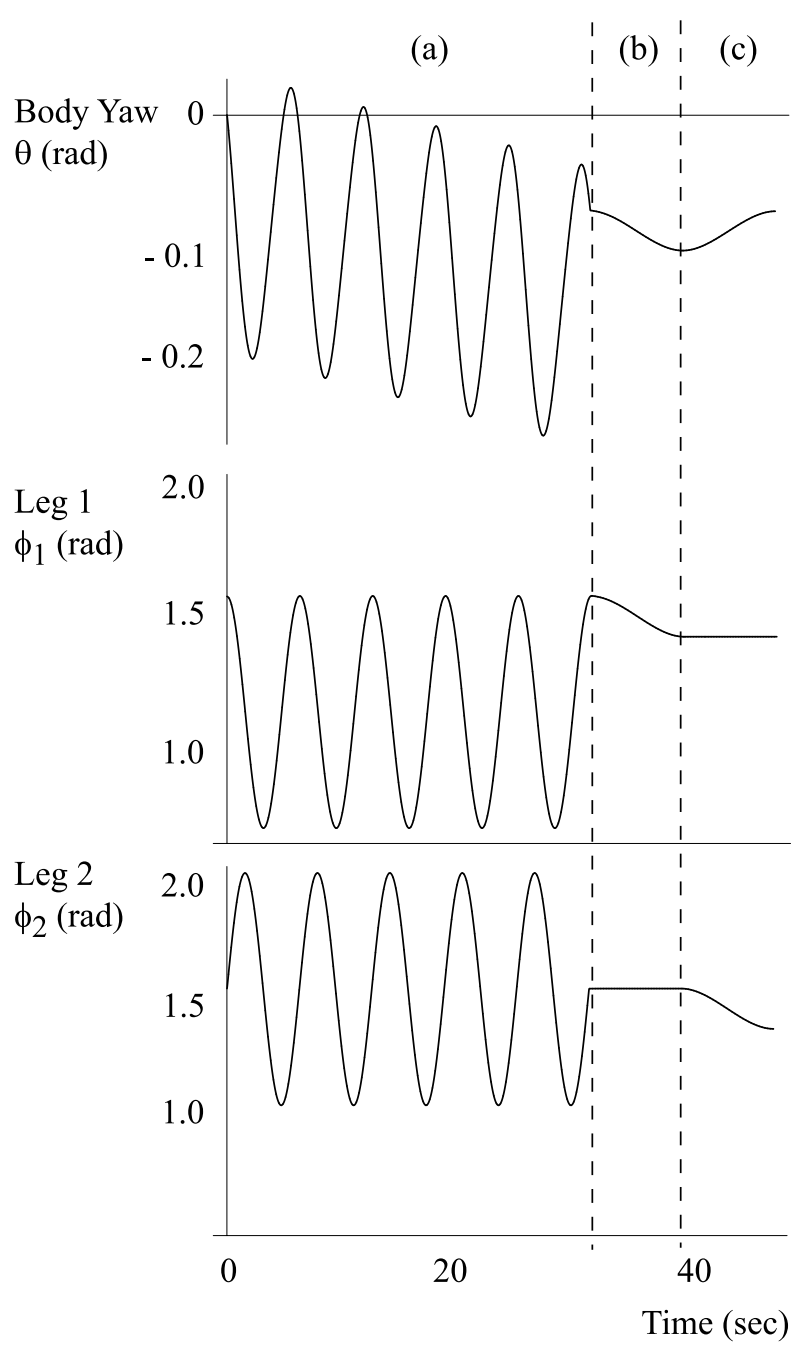

Fig. 4. Time history of body $\mathrm{Z}$ and leg confi gurations while executing a plan to go from start confi guration $q_{s}=(0, \pi / 2, \pi / 2)$ to goal confi guration $q_{g}=(-4 \pi / 180,4 \pi / 9,4 \pi / 9)$ (four degrees body yaw and ten degrees off the legs-up confi guration). Gait 1 is repeated fi ve times in period (a), leg 1 is moved using Gait 2 into its goal position in period (b), and leg 2 is moved using Gait 2 into its goal position in period (c).

leg configuration when executing the plan on the mechanical system using a PD controller (constants $K_{p}=20$ and $\left.K_{D}=10\right)$.

\section{Discussion}

The key contribution of this paper is finding a kinematic reduction of a mechanical system by identifying the system symmetry. Once we deduce the kinematic equations, the planning and control for the mechanical system becomes intuitive. We have only discussed the procedure for the simple Yaw model; we hope this technique of using symmetries to develop kinematic reductions will lead us to a general strategy for understanding and finding motion primitives for complex systems such as the legless 
locomoting RRRobot [4]. Yaw rotations are a crucial component of RRRobot's locomotion, and, with a suitable choice of coordinates, RRRobot's Lagrangian is invariant to yaw rotations. Exploiting this symmetry and developing kinematic equations of motion for at least some of RRRobot's freedoms may help develop RRRobot motion primitives and help plan paths for RRRobot.

\section{CONCLuSion}

We presented controllability and kinematic reduction for a variable inertia mechanical system with drift. We used the symmetry of angular momentum conservation to develop the kinematic representation. Future work will include developing reduced models for systems like RRRobot and generalize these techniques to automatically produce gaits.

\section{ACKNOWLEDGMENT}

This work was supported under NSF IIS 0082339, NSF IIS 0222875, and DARPA/ONR N00014-981-0747 contracts. Matt Mason and George Kantor provided valuable intuition into mechanical systems and feedback on this paper, and Elie Shammas gave useful comments on this paper.

\section{REFERENCES}

[1] R. Abraham and J.E.Marsden. Foundations of Mechanics. Reading, MA: Addison-Wesley, 1978.

[2] R. Balasubramanian, A. A. Rizzi, and M. T. Mason. Legless locomotion for legged robots. In Proceedings of the International Conference on Robots and Intelligent Systems, volume 1, pages 880-885, 2003.

[3] R. Balasubramanian, A. A. Rizzi, and M. T. Mason. Legless locomotion for legged robots. Technical report, Robotics Institute, Carnegie Mellon University, 2004.

[4] R. Balasubramanian, A. A. Rizzi, and M. T. Mason. Legless locomotion: Models and experimental demonstration. In Proceedings of the IEEE International Conference on Robotics and Automation, 2004.

[5] A. Bloch, J. Baillieul, P.Crouch, and J. Marsden. Nonholonomic Mechanics and Control. Springer, 2003.

[6] F. Bullo, A. D. Lewis, and K. M. Lynch. Controllable kinematic reductions for mechanical systems: concepts, computational tools, and examples. In Mathematical Theory of Networks and Systems, August 2002.

[7] F. Bullo and K. M. Lynch. Kinematic controllability for decoupled trajectory planning in underactuated mechanical systems. IEEE Transactions on Robotics and Automation, 17(4):402-412, August 2001.

[8] B. Donald, P. Xavier, J. Canny, and J. Reif. Kinodynamic motion planning. Journal of the ACM, 40(5):1048-1066, 1993.

[9] J. C. Latombe. Robot Motion Planning. Kluwer Academic Publishers, 1991.

[10] A. Lewis, J. Ostrowski, R. Murray, and J. Burdick. Nonholonomic mechanics and locomotion: The snakeboard example. In Proceedings of the International Conference on Robotics and Automation, volume 3, pages 2391-2397, 1994.
[11] A. D. Lewis. When is a mechanical control system kinematic? In Proceedings of the IEEE Conference on Decision Control, volume 2, pages 1162-1167, 1999.

[12] A. D. Lewis. Simple mechanical control systems with constraints. IEEE Transactions on Automatic Control, 45(8):1420-1436, 2000.

[13] A. D. Lewis and R. M. Murray. Confi guration controllability of simple mechanical control systems. SIAM Journal on Control and Optimization, 35(3):766-790, 1997.

[14] S.Kobayashi and K. Nomizu. Foundations of Differential Geometry, volume 1 of Interscience Tracts in Pure and Applied Mathematics. New York, NY: Interscience Publishers, 1963. 